\title{
Impacto das alterações das estruturas do sistema estomatognático na deglutição de idosos acamados
}

\author{
Maira Rozenfeld Olchik*, Annelise Ayres ${ }^{\star \star}$, Alana Verza Signorini ${ }^{\star \star \star}$, Leticia Sousa Flores ${ }^{\star \star \star \star ~}$
}

\section{Resumo}

O envelhecimento pode provocar modificações morfofisiológicas causadas pelo acúmulo de danos ao longo da vida. Em relação aos aspectos do sistema estomatognático, observam-se alterações tanto nas estruturas como nas suas funções. Dessa forma, este estudo tem como objetivo descrever a relação entre as alterações das estruturas do sistema sensório-motor oral e as alterações de deglutição em idosos acamados de uma instituição de longa permanência. O estudo foi do tipo transversal. Para avaliação, foi utilizado o Protocolo de Avaliação Clínica no Leito Elisabete Carrara-de-Angelis. Realizou-se a avaliação das estruturas com base na observação e verificação da tonicidade, mobilidade e sensibilidade, assim como a observação das funções a partir do acompanhamento durante as refeições. A amostra foi composta por 39 idosos, sendo $53,8 \%$ (21) do sexo masculino, a média de idade foi de 82,8 anos $( \pm 10,7)$. Em relação à prevalência da classificação da função de deglutição, foi observado que dezesseis idosos $(41,0 \%)$ apresentaram deglutição normal, quatro $(10,3 \%)$ alteração de deglu- tição e dezenove $(48,7)$ disfagia. Verificou-se relação significativa entre sensibilidade, mobilidade e tonicidade de bochecha, sensibilidade de lábios, tônus de língua e mobilidade de mandíbula com disfagia. Além disso, verificou-se relação significativa entre o sexo feminino e a disfagia. O presente estudo demonstrou relação significativa entre alterações de deglutição e as estruturas do sistema estomatognático.

Palavras-chave: Envelhecimento. Instituição de longa permanência para idosos. Sistema estomatognático. Transtornos de deglutição.

\section{Introdução}

No Brasil, estima-se que $40 \%$ das pessoas com 65 anos ou mais necessitarão de cuidados em uma instituição de longa permanência (ILP) durante a sua vida (VILLAS BOAS; FERREIRA, 2007; ROQUE; BOMFIM; CHIARI, 2010;

* Professora adjunta do curso de Fonoaudiologia da Universidade Federal do Rio Grande do Sul. Endereço para correspondência: Av. Ramiro Barcelos, 2.492, CEP 90035-007, Porto Alegre, RS, Brasil. E-mail: mairarozenfeld@hotmail.com

** Fonoaudióloga clínica, Doutoranda do Programa de Pós-Graduação de Ciências da Saúde da Universidade Federal de Ciências da Saúde de Porto Alegre. E-mail: anneliseayres@hotmail.com

**** Fonoaudióloga do Serviço de Fonoaudiologia do Hospital de Clínicas de Porto Alegre. E-mail: alanass@ gmail.com

***** Fonoaudióloga graduada pela Universidade Federal do Rio Grande do Sul. E-mail: leti.sflores@gmail.com

$\rightarrow$ http://dx.doi.org/10.5335/rbceh.v13i2.5673

Recebido em: 05/01/2016. Aceito em: 10/08/2016. 
BENTES; PEDROSO; MACIEL, 2012). Segundo o Censo 2000, os idosos institucionalizados representavam $0,8 \%$ da população idosa brasileira (INSTITUTO BRASILEIRO DE GEOGRAFIA E ESTATÍSTICA, 2010). Dessa forma, observa-se o aumento significativo do número de idosos institucionalizados, uma vez que existe a exigência de assistência constante na vida diária, que faz com que uma parte dos idosos torne-se dependente, incapaz de manter sua autonomia nas atividades do cotidiano (MENEZES; VICENTE, 2007; LISBOA; CHIANCA, 2012).

Dentre as causas dessa dependência, está o envelhecimento. Esse processo pode provocar modificações morfofisiológicas causadas pelo acúmulo de danos ao longo da vida. Essas alterações tendem a interferir nas atividades de vida diária. Quanto aos aspectos do sistema estomatognático, observam-se alterações tanto nas estruturas (envolvendo mandíbula, área oclusal, lábios, língua, bochechas e palato) como nas funções (mastigação, respiração, deglutição, emissão de voz e fala) (LIMA et al., 2009; CARDOSO; BUJES, 2010; FURTADO; FORTE; LEITE, 2011; GOTTLIEB et al., 2011; CASSOL et al., 2012).

As alterações de deglutição apresentam grande risco aos idosos, pois podem ocasionar desnutrição, desidratação e a pneumonia aspirativa, que é uma das principais causas de morbimortalidade nessa população (ROQUE; BOMFIM; CHIARI, 2010; CASSOL et al., 2012; BOMFIM; CHIARI; ROQUE, 2013). Entre os problemas observados estão tosse, engasgo, queda de saturação, dependência para alimentação, ingestão reduzida, consistência inadequada do alimento, oferta rápida pelo cuidador, problemas relacionados à postura e ao posicionamento e perda dentária (ROQUE; BOMFIM; CHIARI, 2010; CASSOL et al., 2012; BOMFIM; CHIARI; ROQUE, 2013).

Chiari, Roque e Bomfim (2010) apontaram a prevalência de alterações de deglutição em uma amostra de idosos institucionalizados igual a $23,3 \%$, considerando as mais prevalentes: os resíduos alimentares após a deglutição, a tosse durante a alimentação e a alteração vocal após a deglutição. Em estudos norte-americanos e europeus, a prevalência de alterações de deglutição foi descrita como sendo de $26,5 \%$ a $87 \%$ nos idosos institucionalizados (SIEBENS et al., 1986; O`LOUGHLIN; SHANLEY, 1998).

Considerando as características elucidadas, a hipótese deste estudo é de que serão evidenciadas alterações estruturais correlacionadas às de deglutição na população. Portanto, o objetivo deste estudo é descrever a relação entre as alterações das estruturas do sistema sensório-motor oral e alterações de deglutição em idosos acamados de uma instituição de longa permanência de Porto Alegre, no Rio Grande do Sul.

\section{Materiais e métodos}

O estudo realizado é do tipo transversal. O projeto foi aprovado sob o número 19.921 pelo Comitê de Ética e Pesquisa da Universidade Federal do Rio Grande do Sul, instituição responsável pela pesquisa. As avaliações foram realizadas no período de agosto a novembro de 2012 , com duração média de uma hora. Todos os participantes foram selecionados em 
uma instituição de longa permanência de Porto Alegre, na qual era realizado o estágio do curso de Fonoaudiologia da Universidade Federal do Rio Grande do Sul. O representante legal da instituição assinou o termo de consentimento livre e esclarecido autorizando a participação dos idosos na pesquisa.

Como critério de inclusão na amostra determinou-se que seriam todos os indivíduos acamados, de ambos os sexos, da instituição de longa permanência. Foram excluídos os participantes que não completaram todos os itens da avaliação devido ao comprometimento cognitivo, estabelecido pela impossibilidade de dar respostas a comandos simples, ou que tenham sido hospitalizados durante a avaliação, o que totalizou sete idosos, sendo seis do sexo feminino.

Para a avaliação das estruturas e funções do sistema estomatognático, foi utilizado o Protocolo de Avaliação Clínica no Leito (CARRARA-DE-ANGELIS, 2009), composto por oito partes. Realizou-se avaliação das estruturas (lábios, língua, palato mole, mandíbula, laringe, bochechas e estruturas dentárias) observando-se e verificando-se tonicidade, mobilidade e sensibilidade.

Para avaliação e observação das funções, acompanhou-se o paciente durante as refeições (lanche da tarde e/ou jantar), nas consistências prescritas no prontuário de cada paciente (que variavam de sólida, semissólida a pastosa). Para a deglutição, observou-se tempo de trânsito oral, qualidade vocal pós-deglutição, tosse e engasgo. Os itens foram classificados como normal ou alterado. A função foi classificada em normal, alterada ou disfágica.

Para a análise estatística dos dados, realizou-se o cruzamento entre cada item da avaliação estrutural com a função de deglutição por meio do Teste Qui-Quadrado. Para o cruzamento da variável idade com a função de deglutição, utilizou-se o ANOVA. O nível de significância considerado nas análises foi de 5\%. O programa estatístico utilizado foi o Statistical Package for the Social Sciences, versão 18.0.

\section{Resultados}

A amostra foi composta por 39 idosos, sendo 53,8\% (21) do sexo masculino, com média de idade de 82,8 anos $( \pm 10,7)$.

Em relação à prevalência da classificação da função de deglutição, foi observado que dezesseis idosos $(41,0 \%)$ apresentaram deglutição normal, quatro $(10,3 \%)$ alteração de deglutição e dezenove $(48,7 \%)$ disfagia.

A média de idade, em anos, em cada grupo de acordo com função de deglutição foi de $82,7( \pm 10,9)$ para função normal, $77,6( \pm 6,5)$ para deglutição alterada e $83,7( \pm 11,3)$ disfagia, com $\mathrm{p}=0,674$.

Realizou-se o cruzamento entre cada item da avaliação das estruturas do sistema estomatognático com a função de deglutição, conforme exposto na Tabela 1. Verificou-se relação significativa entre sensibilidade, mobilidade e tonicidade de bochecha, sensibilidade de lábios, tônus de língua e mobilidade de mandíbula com disfagia. Além disso, verificou-se relação significativa entre sexo feminino e disfagia. 
Tabela 1 - Correlação entre alterações estruturais e deglutição

\begin{tabular}{|c|c|c|c|c|c|c|c|c|}
\hline & & \multirow{2}{*}{\multicolumn{2}{|c|}{$\begin{array}{c}\text { Deglutição } \\
\text { normal }\end{array}$}} & \multirow{2}{*}{\multicolumn{2}{|c|}{$\begin{array}{c}\text { Alteração de } \\
\text { deglutição }\end{array}$}} & & & \\
\hline & & & & & & \multicolumn{2}{|c|}{ Disfagia } & \multirow[t]{2}{*}{$\mathrm{p}$} \\
\hline & & $\mathrm{n}$ & $\%$ & $\mathrm{n}$ & $\%$ & $\mathrm{n}$ & $\%$ & \\
\hline \multirow{2}{*}{ Bochecha assimetria } & Normal & 11 & 28,2 & 2 & 5,1 & 8 & 20,5 & \multirow{2}{*}{0,204} \\
\hline & Alterado & 4 & 10,2 & 2 & 5,1 & 11 & 28,2 & \\
\hline \multirow{2}{*}{ Bochecha sensibilidade } & Normal & 15 & 38,4 & 3 & 7,6 & 9 & 23,0 & \multirow{2}{*}{$0,007^{*}$} \\
\hline & Alterado & 0 & 0 & 1 & 2,5 & 7 & 17,9 & \\
\hline \multirow{2}{*}{ Bochecha mobilidade } & Normal & 16 & 41,0 & 1 & 2,5 & 4 & 10,2 & \multirow{2}{*}{$0,000^{*}$} \\
\hline & Alterado & 0 & 0 & 3 & 7,6 & 11 & 28,2 & \\
\hline \multirow{2}{*}{ Bochecha tônus } & Normal & 8 & 20,5 & 0 & 0 & 3 & 7,6 & \multirow{2}{*}{$0,036^{*}$} \\
\hline & Alterado & 7 & 17,9 & 4 & 10,2 & 15 & 38,4 & \\
\hline \multirow{2}{*}{ Lábio sensibilidade } & Normal & 16 & 41,0 & 3 & 7,6 & 12 & 30,7 & \multirow{2}{*}{$0,034^{*}$} \\
\hline & Alterado & 0 & 0 & 1 & 2,5 & 6 & 15,3 & \\
\hline \multirow{2}{*}{ Lábio mobilidade } & Normal & 9 & 23,0 & 1 & 2,5 & 7 & 17,9 & \multirow{2}{*}{0,573} \\
\hline & Alterado & 7 & 17,9 & 3 & 7,6 & 11 & 28,2 & \\
\hline \multirow{2}{*}{ Língua tônus } & Normal & 11 & 28,2 & 1 & 2,5 & 7 & 17,9 & \multirow{2}{*}{0,187} \\
\hline & Alterado & 5 & 12,8 & 3 & 7,6 & 11 & 28,2 & \\
\hline \multirow{2}{*}{ Língua sensibilidade } & Normal & 14 & 35,8 & 3 & 7,6 & 11 & 28,2 & \multirow{2}{*}{0,499} \\
\hline & Alterado & 2 & 5,1 & 1 & 2,5 & 4 & 10,2 & \\
\hline \multirow{2}{*}{ Língua mobilidade } & Normal & 11 & 28,2 & 2 & 5,1 & 6 & 15,3 & \multirow{2}{*}{0,271} \\
\hline & Alterado & 5 & 12,8 & 2 & 5,1 & 9 & 23,0 & \\
\hline \multirow{2}{*}{ Língua tônus } & Normal & 13 & 33,3 & 1 & 2,5 & 5 & 12,8 & \multirow{2}{*}{$0,004^{\star}$} \\
\hline & Alterado & 3 & 7,6 & 3 & 7,6 & 12 & 30,7 & \\
\hline \multirow{2}{*}{ Palato mole mobilidade } & Normal & 14 & 35,8 & 3 & 7,6 & 13 & 33,3 & \multirow{2}{*}{0,596} \\
\hline & Alterado & 2 & 5,1 & 1 & 2,5 & 4 & 10,2 & \\
\hline Palato mole sensibilidade & Normal & 14 & 35,8 & 3 & 7,6 & 13 & 33,3 & 0.647 \\
\hline rala mive sensiominade & Alterado & 2 & 5,1 & 1 & 2,5 & 2 & 5,1 & 0,041 \\
\hline Mandíbula mohilidade & Normal & 14 & 35,8 & 2 & 5,1 & 8 & 20,5 & $0057^{*}$ \\
\hline 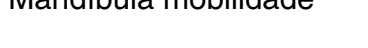 & Alterado & 2 & 5,1 & 2 & 5,1 & 8 & 20,5 & 0,001 \\
\hline Oualidade vocal & Normal & 4 & 10,2 & 1 & 2,5 & 5 & 12,8 & 1000 \\
\hline Qualluaue vulal & Alterado & 11 & 28,2 & 3 & 7,6 & 12 & 30,7 & 1,000 \\
\hline Tosse & Normal & 10 & 25,6 & 3 & 7,6 & 7 & 17,9 & 0,599 \\
\hline Iosse volumar & Alterado & 5 & 12,8 & 1 & 2,5 & 7 & 17,9 & 0,599 \\
\hline
\end{tabular}

Fonte: primária.

Nota: Teste Qui-Quadrado; $* \mathrm{p} \leq 0,05 ; \mathrm{p}=\mathrm{p}$-valor.

A correlação entre sexo e a classificação da deglutição após a avaliação é apresentada na Tabela 2. 
Tabela 2 - Prevalência de sexo na classificação da função deglutição

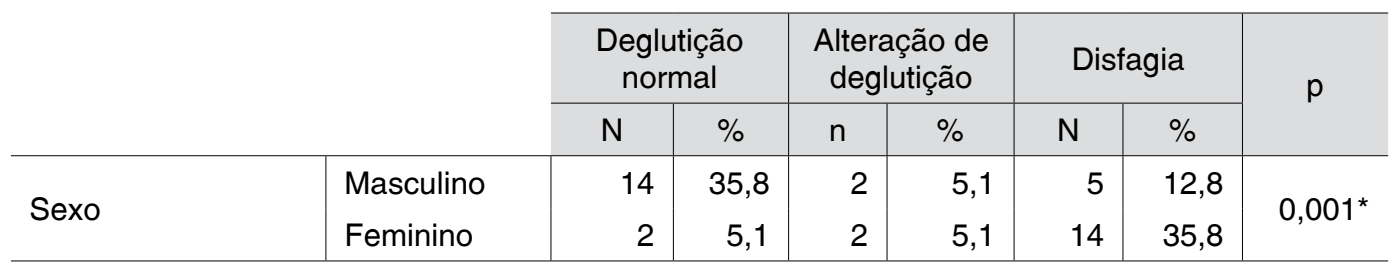

Fonte: primária.

Nota: Teste Qui-Quadrado; * $\mathrm{p} \leq 0,05 ; \mathrm{p}=\mathrm{p}$-valor.

Dessa forma, verifica-se que houve relação significativa entre sexo e classificação de deglutição, demonstrando que idosos do sexo feminino apresentaram maior prevalência de disfagia quando comparados a idosos do sexo masculino, na amostra estudada.

\section{Discussão}

Neste estudo, verificou-se a predominância de indivíduos do sexo masculino na população estudada $(53,8 \%)$, discordando de outros estudos que descrevem predomínio do sexo feminino. Contudo, esse predomínio deve-se ao fato de terem sido excluídos seis indivíduos do sexo feminino (MENEZES; BACHION, 2008; GUTIERREZ et al., 2009; LÓPEZ et al., 2012; OLIVEIRA; DELGADO; BRESCOVICI, 2014).

Apesar de a maioria ser do sexo masculino, houve prevalência de disfagia no sexo feminino. Este estudo vai ao encontro de outra pesquisa (CAMACHO et al., 2011) que apresenta que dentre os 96 indivíduos com disfagia de uma ILP, sendo 5,40\% da amostra total estudada, $80,80 \%$ eram mulheres.
Pela análise dos dados, verifica-se que a maior parte da amostra do presente estudo apresenta a função de deglutição alterada, com a maior parcela apresentando disfagia. Tais achados corroboram com a literatura, na qual se observa que a prevalência de disfagia em idosos residentes de ILPs varia entre $31 \%$ a $65,9 \%$, variação superior à comunidade geral, de $7 \%$ a $40 \%$ (LIN-CHAN LIN et al., 2002; NOGUEIRA; REIS, 2013; BOMFIM; CHIARI; ROQUE, 2013; LINDROOS et al., 2014).

Nessa população, a disfagia encontra-se frequentemente associada à idade avançada e a comorbidades inerentes ao envelhecimento, como acidente vascular cerebral, dependência, demência, múltiplos diagnósticos, depressão, higiene oral precária e fatores ambientais (BOMFIM; CHIARI; ROQUE, 2013; NOGUEIRA; REIS, 2013). A identificação da disfagia nessa população é necessária para a prevenção e minimização dos impactos que a comorbidade pode causar na vida dos idosos, como restrição de participação, desnutrição, desidratação, aspiração traqueal e pneumonia aspirativa, de modo a buscar-se uma melhor qualidade de vida 
para o idoso minimizando seu nível de dependência e cuidados nas ILPs.

Os resultados deste estudo mostraram relação significativa entre sensibilidade, mobilidade e tonicidade de bochecha, sensibilidade de lábios, tônus de língua e mobilidade de mandíbula com disfagia.

Durante o processo de envelhecimento, ocorrem diversas alterações envolvendo, inclusive, o sistema estomatognático e suas funções. A deglutição é uma função biológica complexa do sistema estomatognático, definido como $o$ ato de engolir, podendo ser modificada com o processo de envelhecimento (FERRAZ, 1996; MARCHESAN, 1999; LIMA, 2007). Em estudo realizado com idosos acima de 65 anos, observou-se a diminuição do volume labial, da língua e uma reabsorção dos alvéolos dentários, a diminuição do palato mole e da conformação da epiglote, que se torna menor e mais angulosa (BILTON, 2000).

\section{Conclusão}

Este estudo demonstra resultados significativos de relação entre alterações de deglutição e estruturas do sistema estomatognático. É importante considerar os aspectos estruturais de risco de alterações de deglutição e disfagia na população de idosos institucionalizados. Além disso, verificou-se que as alterações estruturais apresentaram maior impacto na deglutição do que a idade, na amostra estudada.

\section{Impact of changes in the stomatognathic system structures swallowing bedridd en elderly}

\section{Abstract}

Aging may lead tomorphological and physiological changes caused by the accumulation of damage life long. Regarding aspects of the stomatognathic system is observed changes in both structures and in their functions. Thus this study aims describe a relationship between changes in the structures of the oral sensorimotor system and swallowing disorders in bedridden elderly in a longstayinstitution. The study was to realized cross-sectional. Evaluation was used the "Protocolo de Avaliação Clínica no Leito". It was realized the evaluation of structures through observation and verification of tonus, mobility and sensitivity. As well, observing functions during meals. The sample consisted of 39 elderly, and $53.8 \%$ (21) males, an age of media was 82.8 years ( \pm 10.7). Results: The sample consisted of 39 elderly, $53.8 \%$ (21) males, an media of age was 82.8 years $( \pm 10.7)$. Relationship with the prevalence of swallowing function classification, was observed that 16 elderly $(41.0 \%)$ had normal swallowing, $4(10.3 \%)$ alterated swallowing and 19 $(48.7 \%)$ dysphagia. There was a significant relationship between sensitivity, mobility and tonus of cheek, sensitivity of lips, tongue tonus and jaw mobility with dysphagia. In addition, there was a significant relationship between female and dysphagia. So the present study demonstrated significant results for relationship between swallowing disorders and the stomatognathic system structures ratio.

Keywords: Aging. Deglutition disorders. Homes for the aged. Stomatognathic system. 


\section{Referências}

BENTES, A. C. O.; PEDROSO, J. S.; MACIEL, C. A. B. O idoso nas instituições de longa permanência: uma revisão bibliográfica. Aletheia, Canoas, n. 38-39, p. 196-205, dez. 2012.

BILTON, T. L. Estudo da dinâmica da deglutição e de suas variações com o envelhecimento, através do videoglutoesofagograma, em adultos assintomáticos entre 20 e 86 anos. Tese (Doutorado em Ciências Radiológicas) - Universidade Federal de São Paulo, São Paulo, 2000.

BOMFIM, F. M. S.; CHIARI, B. M.; ROQUE, F. P. Fatores associados a sinais sugestivos de disfagia orofaríngea em idosas institucionalizadas. CoDAS, São Paulo, v. 25, n. 2, p. 154-163, jan. 2013.

CAMACHO, M. J. T. et al. Disfagia en ancianos que viven en residencias geriátricas de Barcelona. Gerokomos, Madrid, v. 22, n. 1, p. 20-24, marzo 2011.

CARDOSO, M. C. A. F.; BUJES, R. V. A saúde bucal e as funções de mastigação e deglutição nos idosos. Estudos Interdisciplinares sobre o Envelhecimento, Porto Alegre, v. 1, n. 15, p. 53-67, 2010.

CARRARA-DE-ANGELIS, E. Protocolo de Avaliação Clínica no Leito. In: JOTZ, G. P.; CARRARA-DE-ANGELIS, E.; BARROS, A. P. B. (Org.). Tratado de deglutição e disfagia: no adulto e na criança. Rio de Janeiro: Revinter, 2009. p. 205-207. v. 1.

CASSOL, K. et al. Qualidade de vida em deglutição em idosos saudáveis. Jornal da Sociedade Brasileira de Fonoaudiologia, São Paulo, v. 24, n. 3, p. 223-232, 2012.

FERRAZ, M. C. Manual prático de deglutição atípica e problemas correlatos. Rio de Janeiro: Revinter, 1996.

FURTADO, D. G.; FORTE, F. D. S.; LEITE, D. F. B. M. Uso e necessidade de próteses em idosos: reflexos na qualidade de vida. $R e$ - vista Brasileira de Ciências da Saúde, João Pessoa, v. 15, n. 2, p. 183-190, abr.jun. 2011.

GOTTLIEB, M. G. V. et al. Envelhecimento e longevidade no Rio Grande do Sul: um perfil histórico, étnico e de morbi-mortalidade dos idosos. Revista Brasileira de Geriatria. e Gerontologia, Rio de Janeiro, v. 14, n. 2, p. 365-380, 2011.

GUTIERREZ, S. M. et al. Queixas fonoaudiológicas de idosos residentes em uma instituição de longa permanência. Distúrbios da Comunicação, São Paulo, v. 21, n. 1, p. 21-30, 2009.

INSTITUTO BRASILEIRO DE GEOGRAFIA E ESTATÍSTICA. Síntese de indicadores sociais uma análise das condições de vida da população brasileira, 2010. Censo Demográfico, 2010. Rio de Janeiro: IBGE, 2010.

LI-CHAN LIN, R. N. et al. Prevalence of impaired swallowing in institutionalized older people in Taiwan. Journal of the American Geriatrics Society, New York, v. 50, n. 6, p. 1118-1123, June 2002.

LIMA, L. K. Efeitos do envelhecimento sobre a função de deglutição. In: BUSNELLO, F. M. Aspectos nutricionais no processo do envelhecimento. São Paulo: Atheneu, 2007. Cap. 22. p. 203-210.

LIMA, R. M. F. et al. Adaptações na mastigação, deglutição e fonoarticulação em idosos de instituição de longa permanência. Revista Cefac, São Paulo, v. 11, supl. 3, p. 405-422, 2009.

LINDROOS, E. et al. Caregiver-reported swallowing difficulties, malnutrition, and mortality among older people in assisted living facilities. The Journal of Nutrition, Health \& Aging, Paris, v. 18, n. 7, p. 718-722, July 2014.

LISBOA, C. R.; CHIANCA, T. C. M. Perfil epidemiológico, clínico e de independência funcional de uma população idosa institucionalizada. Revista Brasileira de Enfermagem, Brasília, v. 65 , n. 3, p. 482-487, maio/ jun. 2012. 
LÓPEZ, M. I. F. et al. Detección de disfagia en mayores Institucionalizados. Revista Española de Geriatría y Gerontología, Madrid, v. 47, n. 4, p. 139-140, jul./ago. 2012.

MARCHESAN, I. Q. Deglutição - normalidade. In: FURKIM, A. M.; CELIA, S. S. Disfagias orofaríngeas. Carapicuíba: Pró-Fono Departamento Editorial, 1999. p. 3-18.

MENEZES, R. L. de; BACHION, M. M. Estudo da presença de fatores de riscos intrínsecos para quedas, em idosos institucionalizados. Ciência \&. Saúde Coletiva, Rio de Janeiro, v. 13, n. 4, p. 1.209-1.218, jul./ ago. 2008.

MENEZES, L. N. de; VICENTE, L. C. C. Envelhecimento vocal em idosos institucionalizados. Revista Cefac, São Paulo, v. 9, n. 1, p. 90-98, jan./mar. 2007.

NOGUEIRA, D.; REIS, E. Swallowing disorders in nursing home residents: how can the problem be explained? Clinical Interventions in Aging, Auckland, v. 13, n. 8, p. 221-227, Feb. 2013

OLIVEIRA, B. S. de; DELGADO, S. E.; BRESCOVICI, S. M. Alterações das funções de mastigação e deglutição no processo de alimentação de idosos institucionalizados. $R e$ vista Brasileira de Geriatria e Gerontologia, Rio de Janeiro, v. 17, n. 3, p. 575-587, 2014.

O'LOUGHLIN, G.; SHANLEY, C. Swallowing problems in the nursing hame: a training response. Dysphagia, New York, v. 13, n. 3, p. 172-183, 1998.

ROQUE, F. P.; BOMFIM, F. M. S.; CHIARI, B. M. Descrição da dinâmica de alimentação de idosas institucionalizadas. Revista $d a$ Sociedade Brasileira de Fonoaudiologia, São Paulo, v. 15, n. 2, p. 256-263, 2010.

SIEBENS, H. et al. Correlates and consequences of eating dependency in institutionalized elderly. Journal of the American Geriatrics Society, New York, v. 34, n. 3, p. 192-198, Mar. 1986.
VILLAS BOAS, P. J. F.; FERREIRA, A. L. A. Infecção em idosos internados em instituição de longa permanência. Revista da Associação Medica Brasileira, São Paulo, v. 53, n. 2, p. 126-129, abr. 2007. 